\title{
Integration of DCE-MRI and DW-MRI Quantitative Parameters for Breast Lesion Classification
}

\author{
Roberta Fusco, ${ }^{1}$ Mario Sansone, ${ }^{2}$ Salvatore Filice, ${ }^{1}$ Vincenza Granata, ${ }^{1}$ \\ Orlando Catalano, ${ }^{1}$ Daniela Maria Amato, ${ }^{1}$ Maurizio Di Bonito, ${ }^{3}$ Massimiliano D'Aiuto, ${ }^{4}$ \\ Immacolata Capasso, ${ }^{4}$ Massimo Rinaldo, ${ }^{4}$ and Antonella Petrillo ${ }^{1}$
}

\author{
${ }^{1}$ Department of Diagnostic Imaging, Radiant and Metabolic Therapy, "Istituto Nazionale Tumori Fondazione Giovanni Pascale, \\ IRCCS", Via Mariano Semmola, 80131 Naples, Italy \\ ${ }^{2}$ Department of Electrical Engineering and Information Technologies, University "Federico II" of Naples, Via Claudio 21, \\ 80125 Naples, Italy \\ ${ }^{3}$ Department of Diagnostic and Laboratory Pathology, "Istituto Nazionale Tumori Fondazione Giovanni Pascale, IRCCS", \\ Via Mariano Semmola, 80131 Naples, Italy \\ ${ }^{4}$ Department of Senology, "Istituto Nazionale Tumori Fondazione Giovanni Pascale, IRCCS”, Via Mariano Semmola, \\ 80131 Naples, Italy
}

Correspondence should be addressed to Antonella Petrillo; a.petrillo@istitutotumori.na.it

Received 25 May 2014; Accepted 15 April 2015

Academic Editor: Vivian Barak

Copyright (C) 2015 Roberta Fusco et al. This is an open access article distributed under the Creative Commons Attribution License, which permits unrestricted use, distribution, and reproduction in any medium, provided the original work is properly cited.

Objective. The purpose of our study was to evaluate the diagnostic value of an imaging protocol combining dynamic contrastenhanced MRI (DCE-MRI) and diffusion-weighted MRI (DW-MRI) in patients with suspicious breast lesions. Materials and Methods. A total of 31 breast lesions ( 15 malignant and 16 benign proved by histological examination) in 26 female patients were included in this study. For both DCE-MRI and DW-MRI model free and model based parameters were computed pixel by pixel on manually segmented ROIs. Statistical procedures included conventional linear analysis and more advanced techniques for classification of lesions in benign and malignant. Results. Our findings indicated no strong correlation between DCE-MRI and DWMRI parameters. Results of classification analysis show that combining of DCE parameters or DW-MRI parameter, in comparison of single feature, does not yield a dramatic improvement of sensitivity and specificity of the two techniques alone. The best performance was obtained considering a full combination of all features. Moreover, the classification results combining all features are dominated by DCE-MRI features alone. Conclusion. The combination of DWI and DCE-MRI does not show a potential to dramatically increase the sensitivity and specificity of breast MRI. DCE-MRI alone gave the same performance as in combination with DW-MRI.

\section{Introduction}

Magnetic resonance imaging (MRI) applications such as dynamic contrast enhanced (DCE) and diffusion weighted imaging (DWI) have the potential to provide noninvasive digital biomarkers with good spatial resolution and reproducibility suitable for early detection of breast cancer and for therapy evaluation [1-9]. In general, DCE-MRI has shown high sensitivity for breast cancer detection (89-100\%) [1-12], while DWI has shown utility in predicting suitable therapies and monitoring response [13].
DCE-MRI consists in the serial acquisition of images before and after the injection of intravenous contrast agent; it has been shown to give information about vascular permeability within the tumor $[10,11]$. Different methods for DCE-MRI data analysis have been proposed, ranging from simple semiquantitative inspection of the time-intensity curves (TICs) to more sophisticated tracer kinetics modeling [14-18]. The different methods were designed to capture the biologically relevant components from the dynamic MR signal and to relate them to the underlying pathophysiological processes taking place in the tissue. In principle, 
TABLE 1: Scan settings.

\begin{tabular}{lccc}
\hline Settings & DCE-MRI & DW-MRI & Units \\
\hline TR/TE/ $\alpha$ & $9,8 / 4,76 / 25$ & $7700 / 129 / 90$ & $\mathrm{~ms} / \mathrm{ms} / \mathrm{deg}$ \\
Pulse sequence & T1-weighted 3D FLASH & T2-weighted SPAIR & Axial \\
Plane & Coronal & $183 \times 360$ & $\mathrm{~mm}^{2}$ \\
FOV & $185 \times 370$ & $120 \times 236$ & $\mathrm{pixel}$ \\
Matrix size & $128 \times 256$ & $1.52 \times 1.52$ & $\mathrm{~mm}^{2}$ \\
Pixel spacing & $1.44 \times 1.44$ & 4 & $\mathrm{~mm}$ \\
Slice thickness & 2 & 2 & $\mathrm{~mm}$ \\
Gap between slices & 0 & 24 & - \\
Number of slices & 80 & & \\
\hline
\end{tabular}

the derivation of full-quantitative physiological data from DCE-MRI should rely on the application of appropriate tracer kinetics models to describe the distribution of contrast media following its systemic administration. However, the application of these techniques is still complex and they could not be widely available outside specialist centers. In response to this, many semiquantitative approaches for the classification of TIC shapes have been described and are now in relatively common use in clinical settings [18-26].

DW-MRI images are sensitive to water diffusion; preclinical and clinical data showed that it can reflect vessels structure $[12,13]$. An approximated quantitative analysis of DW-MRI can be performed calculating the apparent diffusion coefficient (ADC) based on the relative signal intensity change of the tissue with increasing $b$ values (see Section 2 ) or by using intravoxel incoherent motion (IVIM) [27-29] modeling for a more accurate quantitative analysis that has the potential to provide information about both the cellularity and perfusion of tumors. With the increasing awareness of the toxicity of MR contrast agents, DW-MRI could be considered a favorable alternative for deriving perfusion information without contrast agent injection [27-33].

At the time of writing only a few studies explored the correlation between these two methods and attempted a comparison in the case of breast cancer; moreover, it could be useful to assess their independence or complementarity.

The objective of the present study is to evaluate the correlation between DCE-MRI and DW-MRI data in breast cancer; moreover, we tried to establish if opportunely combining DCE and DW-MRI features for differentiation of benign and malignant breast lesions could improve performance.

\section{Materials and Methods}

2.1. Patients Characteristics. 31 breast lesions (15 malignant and 16 benign, proved by histological examination) in 26 female patients (mean age $37.2 \pm 10.4$ years, range $14-53$ years) were included in this study. The malignant lesions included 9 infiltrating ductal carcinomas, 3 infiltrating ductal-lobular carcinomas, 1 infiltrating lobular carcinoma, and 2 ductal carcinomas in situ (DCIS). The benign lesions included 11 fibroadenomas and 5 fibrocystic dysplasias.

2.2. MR Protocol. Per each subject, DW-MRI and DCE-MRI data were acquired consecutively during the same session with a 1.5 T scanner (Magnetom Symphony, Siemens Medical System, Erlangen, Germany) equipped with breast dedicated coil. Scan settings are reported in Table 1.

DW-MRI data comprised 7 scans, each corresponding to a different $b$ value $\left(0,50,100,150,400,800\right.$ and $\left.1000 \mathrm{~s} / \mathrm{mm}^{2}\right)$.

DCE-MRI data comprised 10 consecutive scans acquired with an interval between two successive scans of $56 \mathrm{~s}$. The contrast agent bolus, $0.1 \mathrm{~mL} / \mathrm{kg}$ body weight of Gd-DOTA (Dotarem,Guerbet, Roissy CdG Cedex, France), was injected at the start of the first postcontrast scan. An automatic injection system was used (Spectris Solaris EP MR, MEDRAD, Inc., Indianola, PA). The injection flow rate was $2 \mathrm{~mL} / \mathrm{s}$ followed by a flush of $10 \mathrm{~mL}$ saline solution at the same rate.

2.3. Volumes Coregistration. A 3D linear interpolation was performed in order to align DCE and DW data on a common grid. Before alignment the voxel size of DCE and DW was $1.44 \times 1.44 \times 2 \mathrm{~mm}^{3}$ and $1.52 \times 1.52 \times 6 \mathrm{~mm}^{3}$, respectively. After the alignment the common spatial resolution was $1.5 \times$ $1.5 \times 6 \mathrm{~mm}$. For the subsequent analysis only voxels included in both datasets were considered.

2.4. Region of Interest. Region of interests (ROIs) have been manually drawn by an expert radiologist on DCE images with virtual "fat-suppression" obtained subtracting the precontrast from the 5th postcontrast image. Per each patient only the slices including the lesion have been used. Voxels within ROIs were extracted from both DCE and DW realigned volume data. Features from DCE data and DW data have been computed.

2.5. DCE-MRI Features. Per each voxel, 20 features were extracted from DCE data: 17 were model free and 3 were model based.

2.5.1. Model Based Features. The time-course of contrast medium concentration is typically modelled using the extended Tofts model $[22,24]$ :

$$
C_{t}\left(t, K_{\text {trans }}, k_{\mathrm{ep}}\right)=C_{p}(t) \otimes K_{\text {trans }} \cdot e^{-k_{\mathrm{ep}} \cdot t}+v_{p} \cdot C_{p}(t),
$$

where $C_{t}(t)$ is the concentration of contrast medium within the tissue (voxel) of interest; $C_{p}(t)$ is the concentration of contrast medium within the plasma (also called arterial input function (AIF)); $K_{\text {trans }}$ is the volume transfer constant 


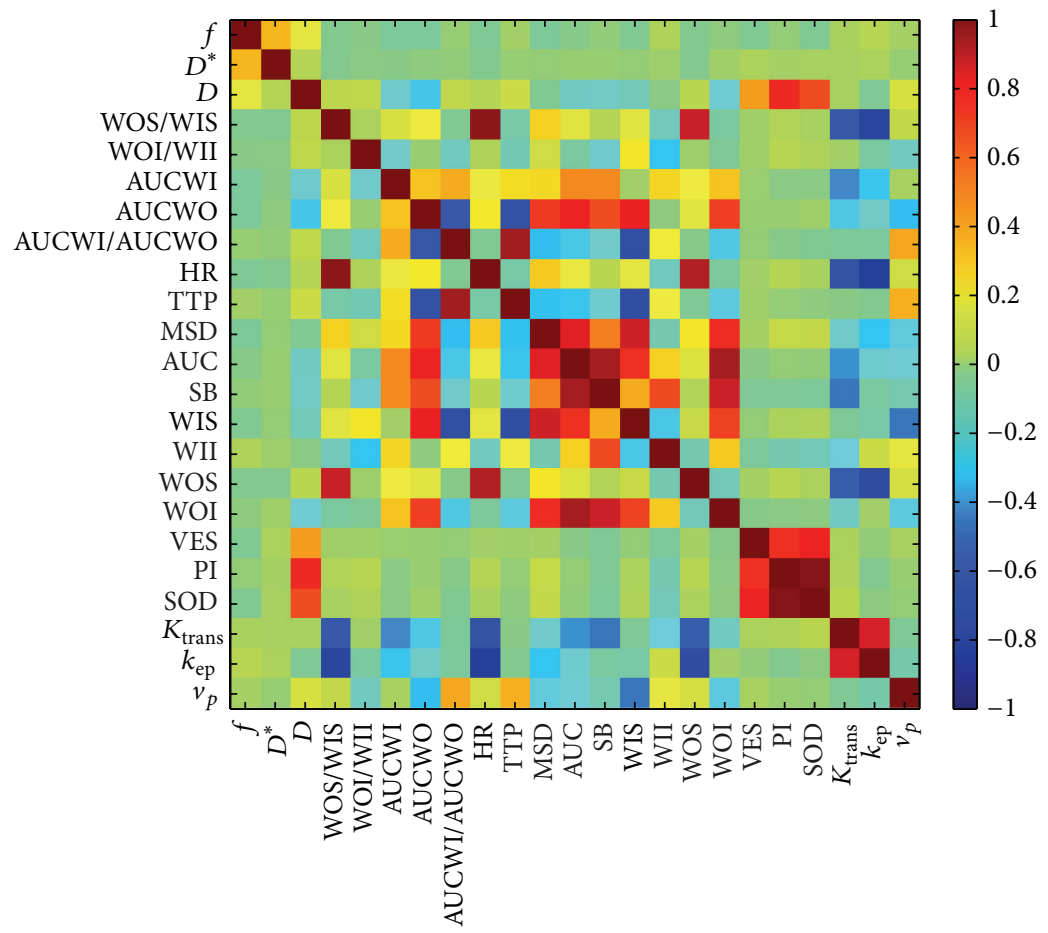

FIGURE 1: Per each couple of features the Spearman correlation coefficient $(r)$ at a voxel-by-voxel level is reported in color code. Yellow to red colors indicate a positive correlation; cyan to blue colors indicate negative correlation. Most of DCE features are not correlated with DW features; a relatively strong positive correlation is observed between $D$ and PI $(r=0.70)$ and between $D$ and SOD $(r=0.60)$.

from plasma to extracellular-extravascular space (EES); $k_{\mathrm{ep}}$ is the diffusion rate constant from EES to plasma; $v_{p}$ is the volume fraction occupied by plasma. These parameters can be related to the level of angiogenic activity. In particular, $K_{\text {trans }}$ represents the vessel permeability and $k_{\text {ep }}$ is linked to the duration of the wash-out phase [21]. We assumed the biexponential AIF proposed by Weinmann et al. [34]:

$$
C_{p}(t)=d\left(a_{1} \exp \left(-m_{1} t\right)+a_{2} \exp \left(-m_{2} t\right)\right),
$$

where $d$ is the administered dose $(\mathrm{mL} / \mathrm{kg}), a_{1}=3.99 \mathrm{~kg} / \mathrm{L}$, $a_{2}=4.78 \mathrm{~kg} / \mathrm{L}, m_{1}=0.144 \mathrm{~min}^{-1}$, and $m_{2}=0.0111 \mathrm{~min}^{-1}$. Contrast medium concentration was calculated from the TIC using the approach suggested by Schabel et al. [35] with a fixed precontrast longitudinal relaxation time, $T_{1,0}$ of $820 \mathrm{~ms}$, appropriate for breast parenchyma.

2.5.2. Model Free Features. For each voxel 17 TIC's shape descriptors were computed using an approach previously reported in [26]: basal signal (SB), maximum signal difference (MSD), the time to peck (TTP), the WI slope (WIS), the WO slope (WOS), the WI intercept (WII), the WO intercept (WOI), the WOS/WIS ratio, and the WOI/WII ratio are under curve (AUC), area under gadolinium curve in the wasin phase (AUCWI), area under gadolinium curve in the washout phase (AUCWO), the AUCWO/AUCWI ratio, and the height ratio (HR).

Moreover in this study the perfusion index (PI) as proposed by [36], the sum of intensity difference (SOD) as proposed by [37], and variance of enhancement slope (VES) as proposed by [38] were calculated.

2.6. $D W-M R I$ Features. Per each voxel, 3 features were extracted from DW data using the Intra Voxel Incoherent Motion (IVIM) model (Figure 1 step 7) [27-29].

DW-MR signal decay is most commonly analyzed using the monoexponential model $[26,27]$ :

$$
\mathrm{ADC}=\frac{\ln \left(S_{0} / S_{b}\right)}{b},
$$

where $S_{b}$ is the MRI signal intensity with diffusion weighting $b, S_{0}$ is the nondiffusion-weighted signal intensity, and ADC is the apparent diffusion coefficient.

For a voxel with a large vascular fraction, the MRI data decay can deviate from a monoexponential form, in particular showing a fast decay in the range of low $b$ values generated by the intravoxel incoherent motion (IVIM) effect $[27,28]$. Thus, in addition to the monoexponential model, a biexponential model was used to estimate the IVIM-related parameters of pseudodiffusivity $\left(D_{p}\right.$ indicated also with $\left.D^{*}\right)$, perfusion fraction $\left(f_{p}\right)$, and tissue diffusivity $(D)$ :

$$
\frac{S_{0}}{S_{b}}=f_{p} \cdot \exp \left(-b \cdot D_{p}\right)+\left(1-f_{p}\right) \cdot \exp (-b \cdot D) \text {. }
$$

The estimation of the three parameters in the biexponential model may often be ill-conditioned because of a limited number of samples, small perfusion fraction, and/or similar 
TABLE 2: Summary of DCE and DW features.

\begin{tabular}{|c|c|c|c|}
\hline ID & Symbol & Description & Units \\
\hline 1 & $f$ & Perfusion fraction & - \\
\hline 2 & $D^{*}$ & Pseudodiffusion coefficient & $\mathrm{mm}^{2} \mathrm{~s}^{-1}$ \\
\hline 3 & $D$ & Tissue diffusion coefficient & $\mathrm{mm}^{2} \mathrm{~s}^{-1}$ \\
\hline 4 & WOS/WIS & Ratio between slopes of wash-out and wash-in phase (see below) & - \\
\hline 5 & WOI/WII & Ratio between intercepts of wash-out and wash-in phase (see below) & - \\
\hline 6 & AUCWI & Area under gadolinium curve in the wash-in phase & s mmol L ${ }^{-1}$ \\
\hline 7 & AUCWO & Area under gadolinium curve in the wash-out phase & $\mathrm{s} \mathrm{mmol} \mathrm{L} \mathrm{L}^{-1}$ \\
\hline 8 & AUCWI/AUCWO & Ratio between areas of wash-out and wash-in phase & - \\
\hline 9 & $\mathrm{HR}$ & Height ratio & - \\
\hline 10 & TTP & Time to peak & $\mathrm{s}$ \\
\hline 11 & MSD & Maximum signal difference & - \\
\hline 12 & AUC & Area under curve & - \\
\hline 13 & SB & Basal signal & - \\
\hline 14 & WIS & Wash-in slope & $\mathrm{s}^{-1}$ \\
\hline 15 & WII & Wash-in intercept & - \\
\hline 16 & WOS & Wash-out slope & $\mathrm{s}^{-1}$ \\
\hline 17 & WOI & Wash-out intercept & - \\
\hline 18 & VES & Variance of enhancement slope & $\mathrm{s}^{-1}$ \\
\hline 19 & PI & Perfusion Index & - \\
\hline 20 & SOD & Sum of intensity difference & - \\
\hline 21 & $K_{\text {trans }}$ & Volume transfer constant from plasma to extracellular-extravascular space & $\mathrm{s}^{-1}$ \\
\hline 22 & $k_{\mathrm{ep}}$ & Diffusion rate constant from extracellular-extravascular space to plasma & $s^{-1}$ \\
\hline 23 & $v_{p}$ & Plasma volume fraction & - \\
\hline
\end{tabular}

compartmental diffusivities, as found in other in vivo IVIM studies [29-33]. Thus, we performed a "two steps" analysis procedure as follows.

Typically, $D_{p}$ is greater than $D[28]$; therefore, when the $b$ value is significantly greater than $\sim 1 / D_{p}$ (e.g., for $D_{p} \sim 10 \mathrm{~mm}^{2} / \mathrm{ms}, b>100 \mathrm{~s} / \mathrm{mm}^{2}$ ), the contribution of the pseudodiffusion term to the signal decay becomes negligible. In this higher $b$ value regime, (3) can be simplified to a monoexponential equation (4), where by $D$ can be estimated:

$$
S_{\text {high }}=S_{0} \cdot \exp (-b \cdot D) \text {. }
$$

Therefore, $D$ is determined from a monoexponential fit to data above a chosen threshold ( $b>200 \mathrm{~s} / \mathrm{mm}^{2}$, in this study). After determining $D$ using (5), $D_{p}, f_{p}$ can be estimated using a nonlinear fit of (4) to the entire dataset that minimizes the residual sum of squares.

2.7. Statistical Analysis. We performed two types of analysis: voxel-by-voxel and lesion-by-lesion analysis. It is expected that the results of the voxel-by-voxel analysis should quantify the possibility to automatically segment images using a combination of DCE and DW information. The results of the lesion-by-lesion analysis should indicate the capability to discriminate benign from malignant. Therefore, both voxel features and lesion features (per each lesion the median value along all the voxels for all features) have been calculated. Table 2 summarizes the 23 features.

In order to assess the correlation between DCE and DWI we made an analysis at a voxel-by-voxel level. Specifically, we computed the Spearman (nonparametric) correlation coefficient between each couple of features. It is expected that strongly correlated (or inversely correlated) features should show a high correlation coefficient (approximately 1 or -1 ).

Subsequently, we used both voxel-by-voxel and lesionby-lesion analysis in order to assess the capability of the features to discriminate benign from malignant voxels or lesions. We applied a Linear Discriminant Analysis (LDA) [39] followed by a linear classifier in order to identify the best combination of features able to produce best classification results. ROC curves for classification were generated. The best linear classifiers were determined by maximizing the area under the ROC curves; best threshold was identified considering the unbalance of benign-malignant lesions [40, 41].

Finally, the performance of a simple algorithm for lesion classification based on voxel-by-voxel analysis was evaluated: a lesion was classified as malignant (benign) if the majority of voxel within that lesion is classified as malignant (benign). Classification based on DCE alone, DW alone, and combination of DCE and DW was compared. In the three cases, the percentage of correctly classified samples was computed for each lesion.

\section{Results}

Figure 1 shows the Spearman correlation coefficient $(r)$ at a voxel-by-voxel level between all feature couples. The numbering of the features is as in Table 2. It can be seen that in 

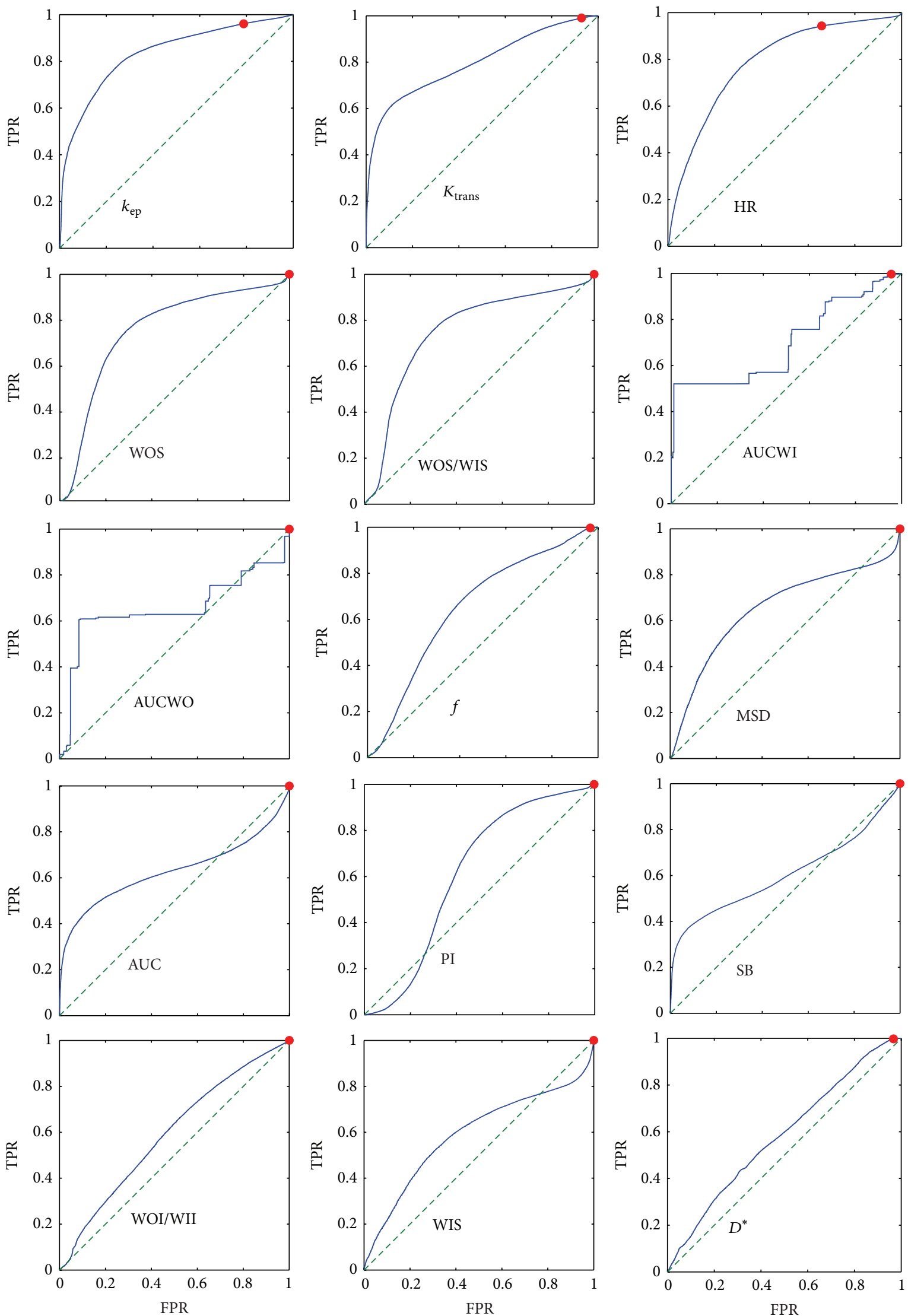

Figure 2: Continued. 

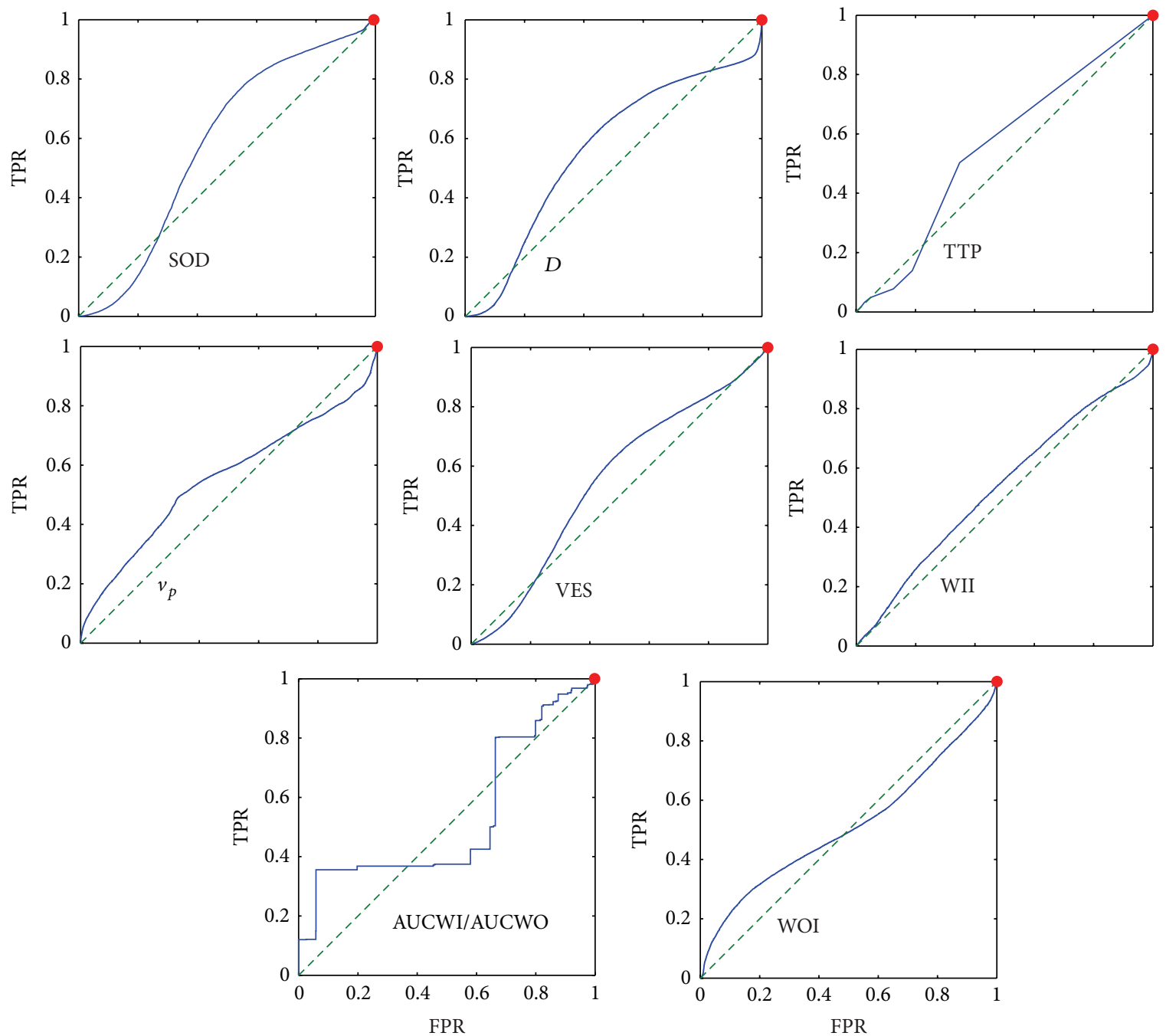

Figure 2: Receiver operating curves (ROCs) of single features in the case of voxel-by-voxel analysis. Per each feature (see Table 2) the ROC is reported in terms of true positive rate (TPR) and false positive rate (FPR). The plots have been aligned according to the area under curve (AUC) in row-wise descending order with the largest AUC at the top-left. The red dot indicates the best compromise between TRP/FPR considering the unbalance between benign-malignant subjects. FPR is generally very high except for $k_{\text {ep }}$ and HR.

general, DCE and IVIM parameters show weak correlation except PI \& $D(r=0.70)$ and SOD \& $D(r=0.60)$.

Figures 2 and 3 show the receiver operating curves (ROCs) for single features in the case of voxel-by-voxel and lesion-by-lesion analysis, respectively. Within a specific ROC the best threshold is indicated by a red dot which has been evaluated considering the unbalance between benign and malignant lesions. The largest area under curve (AUC) with high sensitivity and good specificity has been obtained for $k_{\text {ep }}$, $K_{\text {trans }}$ and HR, in the case of voxel-by-voxel analysis and for $D^{*}$, WIS, and $f$, in the case of lesion-by-lesion analysis (we discarded SB because of very low sensitivity).

Figures 4 and 5 report the results of the best combination of all features in the LDA analysis in the case of voxelby-voxel and lesion-by-lesion analysis respectively. The best threshold is indicated by a red dot, which has been evaluated considering the unbalance between benign and malignant lesions.
Figure 6 shows the percentage of correctly classified samples by Linear Discriminant Analysis for each lesion. Using DCE features only showed the same behavior of combined DCE \& DW. The number of misclassified patients using DCE only and DW only was the same (9 patients were misclassified in both cases).

Tables 3 and 4 report sensitivity and specificity of the parameters in the voxel-by-voxel analysis that provide the maximum area under the ROC (AUROC).

\section{Discussion}

The purpose of our study was to evaluate the diagnostic value of an imaging protocol that combines dynamic contrastenhanced MRI (DCE-MRI) and diffusion-weighted imaging (DWI) in patients with suspicious breast lesions and to determine if additional information provided by DWI could improve the diagnostic value of breast MRI. 

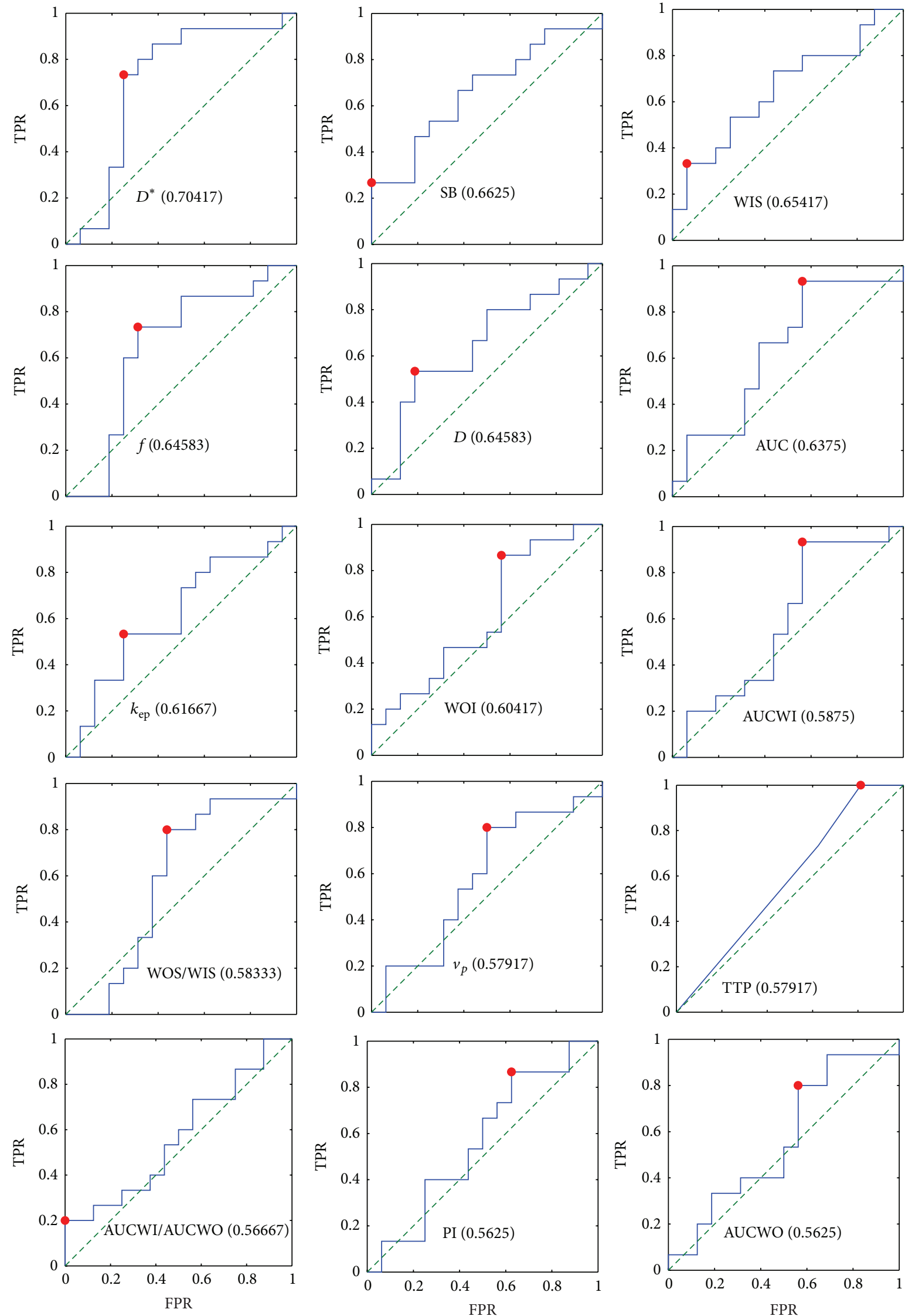

Figure 3: Continued. 

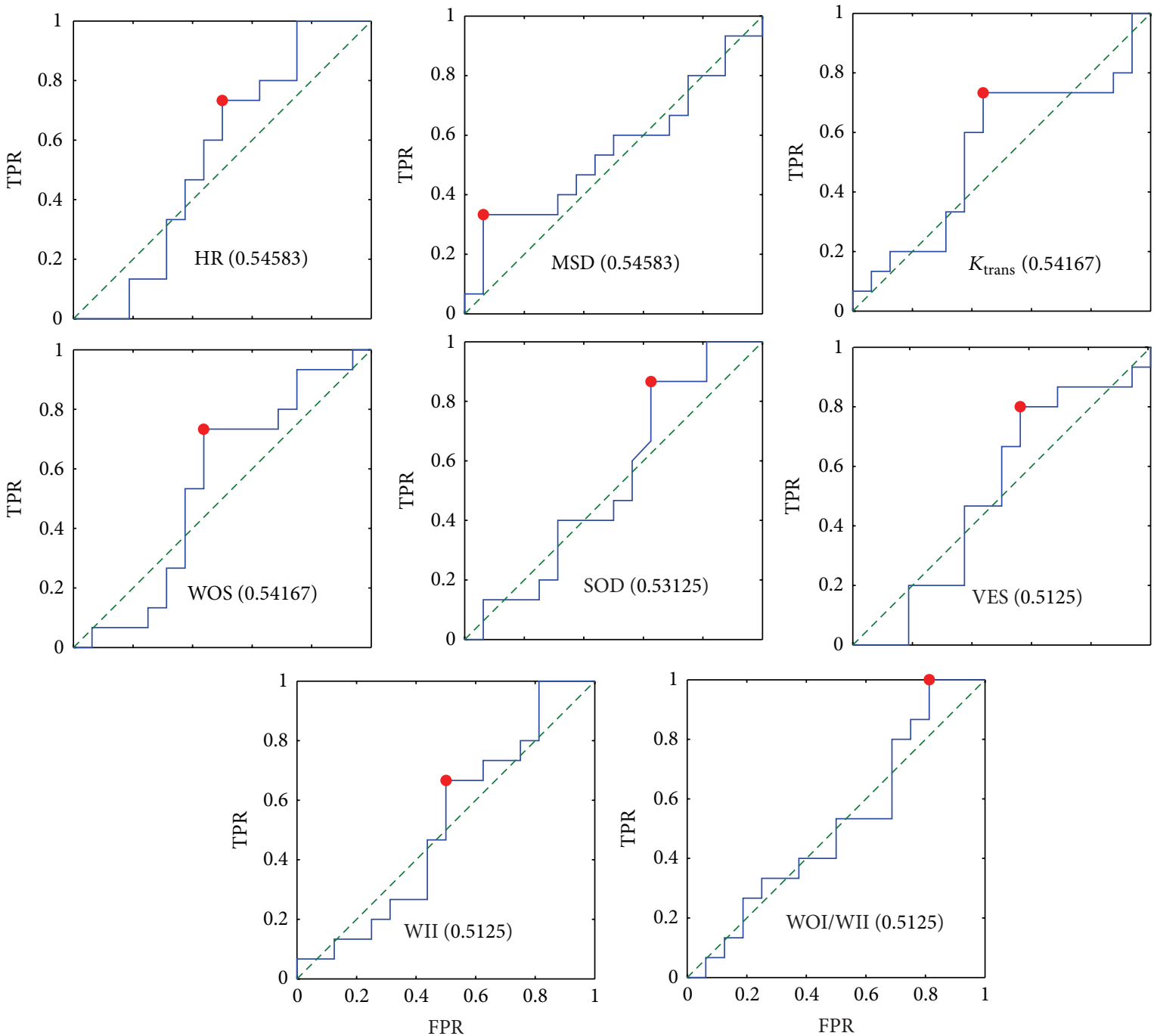

FIGURE 3: Receiver operating curves (ROCs) of single features in the case of lesion-by-lesion analysis. Per each feature (see Table 2) the ROC is reported in terms of true positive rate (TPR) and false positive rate (FPR). The plots have been aligned according to the area under curve (AUC) in row-wise descending order with the largest AUC at the top-left (per each feature the AUC is indicated in parenthesis). The red dot indicates the best value considering the unbalance between benign-malignant subjects.

TABLE 3: Sensitivity and specificity of the parameters in the pixelby-pixel analysis that provide the maximum area under the ROC (AUROC).

\begin{tabular}{lccc}
\hline Parameters & AUROC & Sensitivity & Specificity \\
\hline$k_{\text {ep }}$ & 0.7 & 0.96 & 0.22 \\
$K_{\text {trans }}$ & 0.66 & 0.99 & 0.18 \\
HR & 0.65 & 0.94 & 0.35 \\
\hline
\end{tabular}

Our findings showed that no strong correlation was obtained between DCE-MRI and DW-MRI features (Figure 1). The largest values were obtained in correspondence of the pairs PI \& $D$ and SOD \& $D$, probably because PI and SOD are the only two features that describe the whole trend of the time intensity curve course, which allow obtaining the best discrimination between the different types of lesion.
TABLE 4: Sensitivity and specificity of the parameters in the lesionby-lesion analysis that provide the maximum area under the ROC (AUROC). We have discarded SB because of low sensitivity.

\begin{tabular}{lccc}
\hline Parameters & AUROC & Sensitivity & Specificity \\
\hline$D^{*}$ & 0.7 & 0.73 & 0.75 \\
WIS & 0.65 & 0.33 & 0.94 \\
$f$ & 0.64 & 0.73 & 0.69 \\
\hline
\end{tabular}

The ROC analysis showed that single features (both DCE and DW) do not have a good discriminative power (Figures 2 and 3). Moreover, the unbalance of our data does not allow determining a good threshold with both high sensitivity and specificity.

Results of Linear Discriminant Analysis (Figures 4 and 5) showed that the use of a combination of DCE and DW 


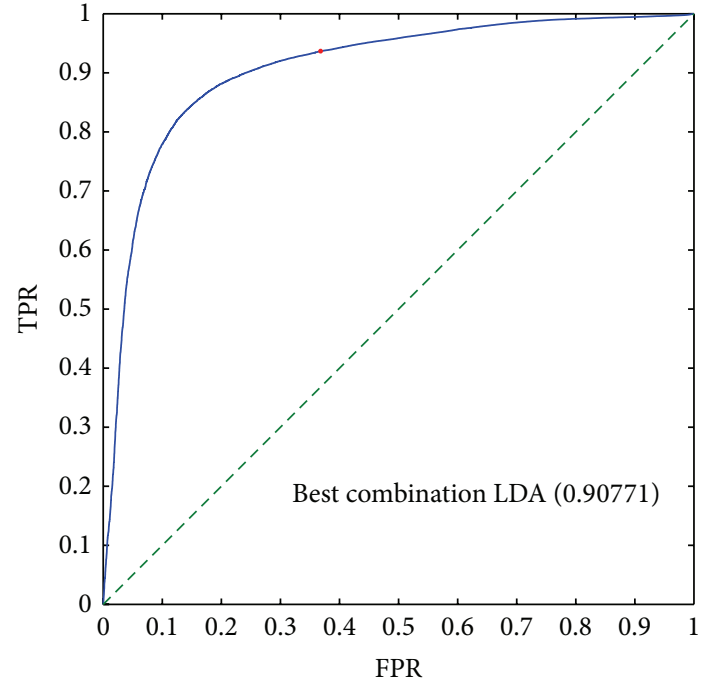

FIGURE 4: ROC analysis of the best linear combination of all features obtained using Linear Discriminant Analysis in the case of pixelby-pixel analysis. The AUC is indicated in parenthesis. The red dot indicates the best point considering the unbalance between benignmalignant patients: the TPR is approximately 0.93 and the FPR is about 0.35 .

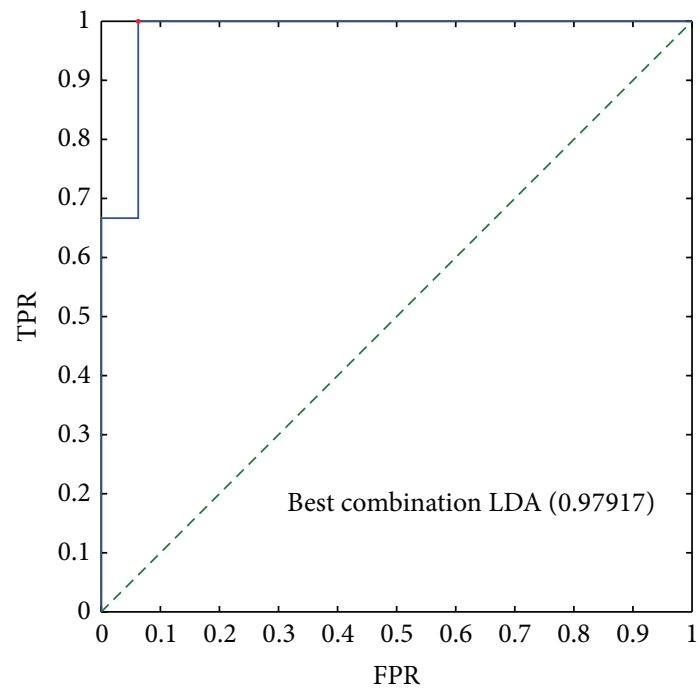

FIGURE 5: ROC analysis of the best linear combination of all features obtained using Linear Discriminant Analysis in the case of lesionby-lesion analysis. The red dot indicates the best point considering the unbalance between benign-malignant patients. The AUC is reported in parenthesis. The TPR is 1 with FPR less than 0.1 .

features, in comparison to single features, has the potential to improve sensitivity and specificity.

However, the potential of both the set of features cannot be achieved using simple classification algorithms such as the one proposed in Section 2.7. Further investigations toward to best way to combine the information from DCE and DW should be performed.

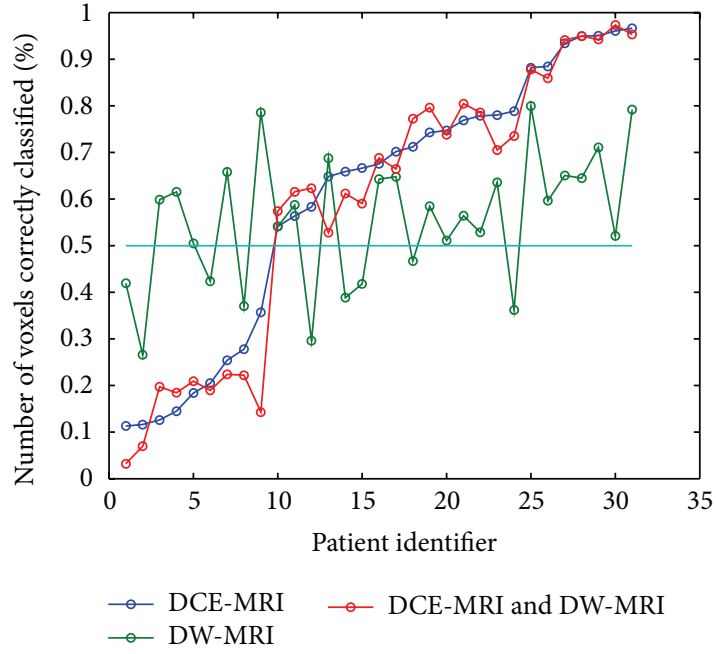

FIGURE 6: Result of a simple algorithm for classifying benign and malignant lesions: the voxels within a lesion can be classified as benign or malignant using the best combination of features in the pixel-by-pixel analysis: a lesion is classified as malignant if it has a percentage of malignant voxels higher than $50 \%$. Per each patient, the percentage of the correctly classified voxels within the ROI is reported: if this percentage is higher than $50 \%$ than the lesion will be correctly identified. It can be seen that using DCE alone (blue line) only 9 lesions have been incorrectly classified. Using only DW we have again 9 lesions misclassified, but they are different from the previous ones. Moreover, the combination of DCE and DW produces the same results as DCE only.

According to our knowledge, no previous study in the literature tried to combine DCE and DW-MRI features including model free and model based parameters evaluated by DCE-MRI data and IVIM parameters evaluated by DWMRI data, after automatic registration and preprocessing of two volumes, to assess the accuracy in differentiation of benign and malignant breast lesions and to evaluate the improvement of additional of DW-MRI parameters to DCEMRI features in breast lesion classification.

Several authors in recent literature have combined DCE and DW-MRI data in breast cancer to different aims. Rahbar et al. [42] developed a model incorporating DCE and DW-MRI features, including semimodel free parameters and $\mathrm{ADC}$, to differentiate high-nuclear-grade (HNG) from non-HNG ductal carcinoma in situ (DCIS) in vivo. Those preliminary findings suggested that DCE and DWMR imaging features may aid in identifying patients with high risk DCIS. Kul et al. [43] evaluated the diagnostic value of an imaging protocol that combined DCE and DW-MRI in patients with suspicious breast lesions and to determine if additional information provided by DWI improves the diagnostic value of breast MRI. They concluded that the combination of DWI and DCE-MRI has the potential to increase the specificity of breast MRI. Partridge et al. [44] showed that ADC can improve the positive predictive value of breast MRI for lesions of varied types and sizes. Jena et al. [45] have tried to evaluate the combined effect of capillary permeability $\left(K_{\text {trans }}\right)$ and tissue cellularity (ADC) on 
the diagnostic accuracy for differentiating benign and malignant breast lesions by incorporating these parameters in routine clinical protocol for breast MRI. Wu et al. [46] reported in their study that the combined use of DW-MRI and CEMRI has the potential to improve the diagnostic performance in monitoring neoadjuvant chemotherapy (NAC). Atuegwu et al. [47] presented a methodology for incorporating ADC and kinetic DCE-MRI features into a simple mathematical model of tumor growth to predict the tumor cellularity and early treatment response at NAC. In this contribution, results indicate how the integration of DW- and DCE-MRI data can improve specificity and positive predictive value to separate responder by nonresponder patients after one cycle of NAC. It is worth mentioning the recent work proposed by Cai et al. [48, 49]: they proposed a machine learning approach to combining diffusion-weighted imaging (DWI), morphology, and kinetic features from DCE-MRI in order to improve the discrimination power of malignant from benign breast masses. They examined seven features divided in four groups: morphological features, texture features, kinetic features, and one DWI feature (apparent diffusion coefficient). Together with the selected diagnostic features, various classical classification schemes were used to test their discrimination power through cross validation scheme. They concluded that multisided variables, which characterize the morphological, kinetic, pathological properties, and DWI measurement of ADC, could improve the discriminatory power of breast lesions.

However, some drawbacks must be underlined: in particular, they used a nonlinear classifier combining the seven features with support vector machine, Bayesian classifier, $k$-nearest neighbours, and logistic regression model, all approaches that determine a nonlinear manipulation of features; this latter is not easy to understand by radiologists with respect to a linear combination of features. Moreover, in our study, in contrast to Cai et al. [48, 49], we combined features including model free and model based parameters evaluated by DCE-MRI data and DW-MRI data.

A few remarks must be made: in our study model based parameters were used because they are more strongly related to physiological characteristics of the tissue (perfusion). We did not considered morphological features, as done in Cai et al. [48, 49], because we were interested only in functional aspects of the lesion: this could explain differences in results between our study and cited studies that included also morphological and textural features.

A major limitation of our study is the small size of the population: an increase is required to increase the power of the statistical tests and to detect statistical differences between the two groups (benign and malignant lesions).

Although not conclusive, our results seem to suggest that the combined use of DCE-MRI and DW-MRI does not provide a dramatic improvement compared to the use of DCE-MRI features alone, in the classification of breast lesions.

\section{Conflict of Interests}

The authors declare that they have no competing interests.

\section{References}

[1] W. Huang, P. R. Fisher, K. Dulaimy, L. A. Tudorica, B. O’Hea, and T. M. Button, "Detection of breast malignancy: diagnostic MR protocol for improved specificity," Radiology, vol. 232, no. 2, pp. 585-591, 2004.

[2] S. H. Heywang-Köbrunner, P. Viehweg, A. Heinig, and C. Küchler, "Contrast-enhanced MRI of the breast: accuracy, value, controversies, solutions," European Journal of Radiology, vol. 24, no. 2, pp. 94-108, 1997.

[3] M. D. Schnall, S. Rosten, S. Englander, S. G. Orel, and L. W. Nunes, "A combined architectural and kinetic interpretation model for breast MR images," Academic Radiology, vol. 8, no. 7, pp. 591-597, 2001.

[4] K. Kinkel, T. H. Helbich, L. J. Esserman et al., "Dynamic highspatial-resolution MR imaging of suspicious breast lesions: diagnostic criteria and interobserver variability," American Journal of Roentgenology, vol. 175, no. 1, pp. 35-43, 2000.

[5] R. M. L. Warren, L. Pointon, D. Thompson et al., "Reading protocol for dynamic contrast-enhanced MR images of the breast: sensitivity and specificity analysis," Radiology, vol. 236, no. 3, pp. 779-788, 2005.

[6] N. H. G. M. Peters, I. H. M. Borel Rinkes, N. P. A. Zuithoff, W. P. T. M. Mali, K. G. M. Moons, and P. H. M. Peeters, "Meta-analysis of MR imaging in the diagnosis of breast lesions," Radiology, vol. 246, no. 1, pp. 116-124, 2008.

[7] L. W. Nunes, M. Schnall, E. S. Siegelman et al., "Diagnostic performance characteristics of architectural features revealed by high spatial-resolution MR imaging of the breast," American Journal of Roentgenology, vol. 169, no. 2, pp. 409-415, 1997.

[8] C. K. Kuhl, P. Mielcareck, S. Klaschik et al., "Dynamic breast MR imaging: are signal intensity time course data useful for differential diagnosis of enhancing lesions?" Radiology, vol. 211, no. 1, pp. 101-110, 1999.

[9] M. C. Lloyd, P. Allam-Nandyala, C. N. Purohit et al., "Using image analysis as a tool for assessment of prognostic and predictive biomarkers for breast cancer: how reliable is it?" Journal of Pathology Informatics, vol. 1, article 29, 2010.

[10] M. V. Knopp, F. L. Giesel, H. Marcos, H. von Tengg-Kobligk, and P. Choyke, "Dynamic contrast-enhanced magnetic resonance imaging in oncology," Topics in Magnetic Resonance Imaging, vol. 12, no. 4, pp. 301-308, 2001.

[11] M. Y. Su, Y. C. Cheung, J. P. Fruehauf et al., "Correlation of dynamic contrast enhancement MRI parameters with microvessel density and VEGF for assessment of angiogenesis in breast cancer," Journal of Magnetic Resonance Imaging, vol. 18, no. 4, pp. 467-477, 2003.

[12] P. Gibbs, G. P. Liney, M. D. Pickles, B. Zelhof, G. Rodrigues, and L. W. Turnbull, "Correlation of ADC and T2 measurements with cell density in prostate cancer at 3.0 Tesla," Investigative Radiology, vol. 44, no. 9, pp. 572-576, 2009.

[13] Y. Matsumoto, M. Kuroda, R. Matsuya et al., "In vitro experimental study of the relationship between the apparent diffusion coefficient and changes in cellularity and cell morphology," Oncology Reports, vol. 22, no. 3, pp. 641-648, 2009.

[14] G. Brix, J. Griebel, F. Kiessling, and F. Wenz, “Tracer kinetic modelling of tumour angiogenesis based on dynamic contrastenhanced CT and MRI measurements," European Journal of Nuclear Medicine and Molecular Imaging, vol. 37, supplement 1, pp. S30-S51, 2010.

[15] S. Sourbron, “Technical aspects of MR perfusion," European Journal of Radiology, vol. 76, no. 3, pp. 304-313, 2010. 
[16] M. Sansone, R. Fusco, A. Petrillo, M. Petrillo, and M. Bracale, "An expectation-maximisation approach for simultaneous pixel classification and tracer kinetic modelling in dynamic contrast enhanced-magnetic resonance imaging," Medical and Biological Engineering and Computing, vol. 49, no. 4, pp. 485-495, 2011.

[17] R. Fusco, M. Sansone, M. Petrillo, and A. Petrillo, "Influence of parameterization on tracer kinetic modeling in DCE-MRI," Journal of Medical and Biological Engineering, vol. 34, no. 2, pp. 157-163, 2014.

[18] R. Fusco, M. Sansone, S. Maffei, N. Raiano, and A. Petrillo, "Dynamic contrast-enhanced MRI in breast cancer: a comparison between distributed and compartmental tracer kinetic models," Journal of Biomedical Graphics and Computing, vol. 2, no. 2, pp. 23-26, 2012.

[19] M. Goto, H. Ito, K. Akazawa et al., "Diagnosis of breast tumors by contrast-enhanced MR imaging: comparison between the diagnostic performance of dynamic enhancement patterns and morphologic features," Journal of Magnetic Resonance Imaging, vol. 25, no. 1, pp. 104-112, 2007.

[20] R. Fusco, S. Filice, V. Granata et al., "Can semi-quantitative evaluation of uncertain (type II) time-intensity curves improve diagnosis in breast DCE-MRI?” Journal of Biomedical Science and Engineering, vol. 6, no. 3, pp. 418-425, 2013.

[21] D. J. Collins and A. R. Padhani, "Dynamic magnetic resonance imaging of tumor perfusion. Approaches and biomedical challenges," IEEE Engineering in Medicine and Biology Magazine, vol. 23, no. 5, pp. 65-83, 2004.

[22] P. S. Tofts, "Modeling tracer kinetics in dynamic Gd-DTPA MR imaging," Journal of Magnetic Resonance Imaging, vol. 7, no. 1, pp. 91-101, 1997.

[23] C. Yang, G. S. Karczmar, M. Medved, and W. M. Stadler, "Estimating the arterial input function using two reference tissues in dynamic contrast-enhanced MRI studies: fundamental concepts and simulations," Magnetic Resonance in Medicine, vol. 52, no. 5, pp. 1110-1117, 2004.

[24] G. Brix, W. Semmler, R. Port, L. R. Schad, G. Layer, and W. J. Lorenz, "Pharmacokinetic parameters in CNS Gd-DTPA enhanced MR imaging," Journal of Computer Assisted Tomography, vol. 15, no. 4, pp. 621-628, 1991.

[25] T. E. Yankeelov, J. J. Luci, M. Lepage et al., "Quantitative pharmacokinetic analysis of DCE-MRI data without an arterial input function: a reference region model," Magnetic Resonance Imaging, vol. 23, no. 4, pp. 519-529, 2005.

[26] R. Fusco, A. Petrillo, M. Petrillo, and M. Sansone, "Use of tracer kinetic models for selection of semi-quantitative features for DCE-MRI data classification," Applied Magnetic Resonance, vol. 44, no. 11, pp. 1311-1324, 2013.

[27] D. Le Bihan, E. Breton, and D. Lallemand, "MR imaging of intravoxel incoherent motions: application to diffusion and perfusion in neurologic disorders," Radiology, vol. 161, no. 2, pp. 401-407, 1986.

[28] D. Le Bihan, E. Breton, D. Lallemand, M.-L. Aubin, J. Vignaud, and M. Laval-Jeantet, "Separation of diffusion and perfusion in intravoxel incoherent motion MR imaging," Radiology, vol. 168, no. 2, pp. 497-505, 1988.

[29] A. Luciani, A. Vignaud, M. Cavet et al., "Liver cirrhosis: intravoxel incoherent motion MR imaging-pilot study," Radiology, vol. 249, no. 3, pp. 891-899, 2008.

[30] R. Wirestam, M. Borg, S. Brockstedt, A. Lindgren, S. Holtås, and F. Ståhlberg, "Perfusion-related parameters in intravoxel incoherent motion MR imaging compared with $\mathrm{CBV}$ and $\mathrm{CBF}$ measured by dynamic susceptibility-contrast MR technique," Acta Radiologica, vol. 42, no. 2, pp. 123-128, 2001.

[31] T. Moteki and H. Horikoshi, "Evaluation of hepatic lesions and hepatic parenchyma using diffusion-weighted echo-planar MR with three values of gradient b-factor," Journal of Magnetic Resonance Imaging, vol. 24, no. 3, pp. 637-645, 2006.

[32] V. Callot, E. Bennett, U. K. M. Decking, R. S. Balaban, and H. Wen, "In vivo study of microcirculation in canine myocardium using the IVIM method," Magnetic Resonance in Medicine, vol. 50, no. 3, pp. 531-540, 2003.

[33] L. Yao and U. Sinha, "Imaging the microcirculatory proton fraction of muscle with diffusion-weighted echo-planar imaging," Academic Radiology, vol. 7, no. 1, pp. 27-32, 2000.

[34] H. J. Weinmann, M. Laniado, and W. Mützel, "Pharmacokinetics of GdDTPA/dimeglumine after intravenous injection into healthy volunteers," Physiological Chemistry and Physics and Medical NMR, vol. 16, no. 2, pp. 167-172, 1984.

[35] M. C. Schabel, G. R. Morrell, K. Y. Oh, C. A. Walczak, R. B. Barlow, and L. A. Neumayer, "Pharmacokinetic mapping for lesion classification in dynamic breast MRI," Journal of Magnetic Resonance Imaging, vol. 31, no. 6, pp. 1371-1378, 2010.

[36] H. L. Sang, H. K. Jong, S. P. Jeong et al., "Texture analysis of lesion perfusion volumes in dynamic contrast-enhanced breast MRI," in Proceedings of the 5th IEEE International Symposium on Biomedical Imaging: From Nano to Macro (ISBI '08), pp. 15451548, Paris, France, May 2008.

[37] T. Twellmann, A. Saalbach, M. O. Leach, and T. W. Nattkemper, "Image fusion for dynamic contrast enhanced magnetic resonance imaging," BioMedical Engineering Online, vol. 3, article $35,2004$.

[38] S. H. Lee, J. H. Kim, N. Cho et al., "Multilevel analysis of spatiotemporal association features for differentiation of tumor enhancement patterns in breast DCE-MRI," Medical Physics, vol. 37, no. 8, pp. 3940-3956, 2010.

[39] W. J. Krzanowski, Principles of Multivariate Analysis: A User's Perspective, Oxford University Press, New York, NY, USA, 1988.

[40] T. Fawcett, "ROC graphs: notes and practical considerations for researchers," HP Labs Tech. Rep., HP Labs, Palo Alto, Calif, USA, 2003.

[41] E. F. Schisterman, N. J. Perkins, A. Liu, and H. Bondell, "Optimal cut-point and its corresponding Youden Index to discriminate individuals using pooled blood samples," Epidemiology, vol. 16, no. 1, pp. 73-81, 2005.

[42] H. Rahbar, S. C. Partridge, W. B. DeMartini et al., "In vivo assessment of ductal carcinoma in situ grade: a model incorporating dynamic contrast-enhanced and diffusion-weighted breast MR imaging parameters," Radiology, vol. 263, no. 2, pp. 374-382, 2012.

[43] S. Kul, A. Cansu, E. Alhan, H. Dinc, G. Gunes, and A. Reis, "Contribution of diffusion-weighted imaging to dynamic contrast-enhanced MRI in the characterization of breast tumors," American Journal of Roentgenology, vol. 196, no. 1, pp. 210-217, 2011.

[44] S. C. Partridge, W. B. DeMartini, B. F. Kurland, P. R. Eby, S. W. White, and C. D. Lehman, "Quantitative diffusion-weighted imaging as an adjunct to conventional breast MRI for improved positive predictive value," American Journal of Roentgenology, vol. 193, no. 6, pp. 1716-1722, 2009.

[45] A. Jena, S. Taneja, and S. B. Mehta, "Integrated quantitative DCE-MRI and DW-MRI to characterize breast lesions," European Journal of Radiology, vol. 81, pp. S64-S65, 2012. 
[46] L.-M. Wu, J.-N. Hu, H.-Y. Gu, J. Hua, J. Chen, and J.-R. Xu, "Can diffusion-weighted MR imaging and contrast-enhanced MR imaging precisely evaluate and predict pathological response to neoadjuvant chemotherapy in patients with breast cancer?" Breast Cancer Research and Treatment, vol. 135, no. 1, pp. 17-28, 2012.

[47] N. C. Atuegwu, L. R. Arlinghaus, X. Li et al., "Parameterizing the logistic model of tumor growth by DW-MRI and DCE-MRI data to predict treatment response and changes in breast cancer cellularity during neoadjuvant chemotherapy," Translational Oncology, vol. 6, no. 3, pp. 256-264, 2013.

[48] H. Cai, L. Liu, Y. Peng, Y. Wu, and L. Li, "Diagnostic assessment by dynamic contrast-enhanced and diffusion-weighted magnetic resonance in differentiation of breast lesions under different imaging protocols," BMC Cancer, vol. 14, no. 1, article 366, 2014.

[49] H. Cai, Y. Peng, C. Ou, M. Chen, and L. Li, "Diagnosis of breast masses from dynamic contrast-enhanced and diffusionweighted MR: a machine learning approach," PLoS ONE, vol. 9, no. 1, Article ID e87387, 2014. 


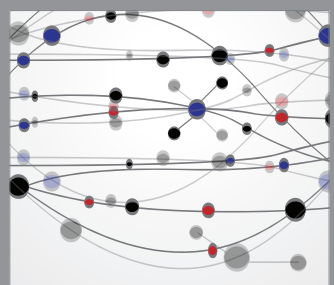

The Scientific World Journal
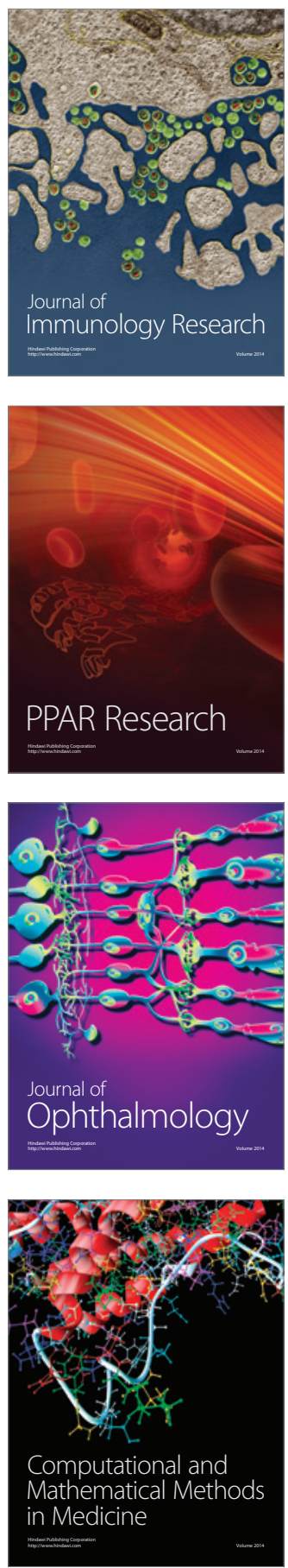

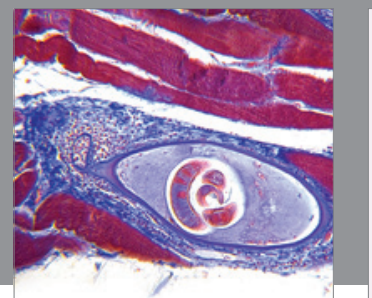

Gastroenterology

Research and Practice
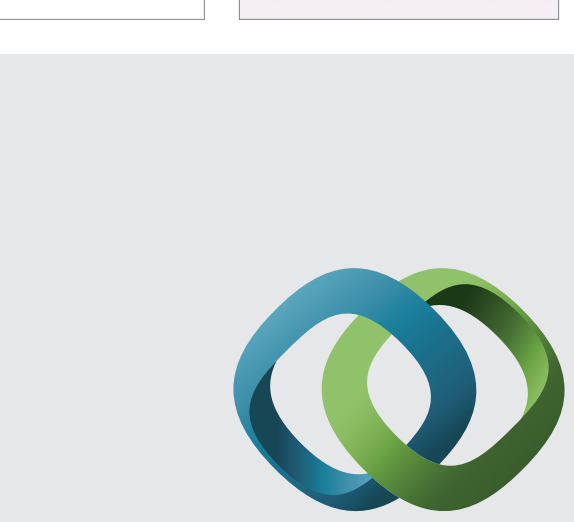

\section{Hindawi}

Submit your manuscripts at

http://www.hindawi.com
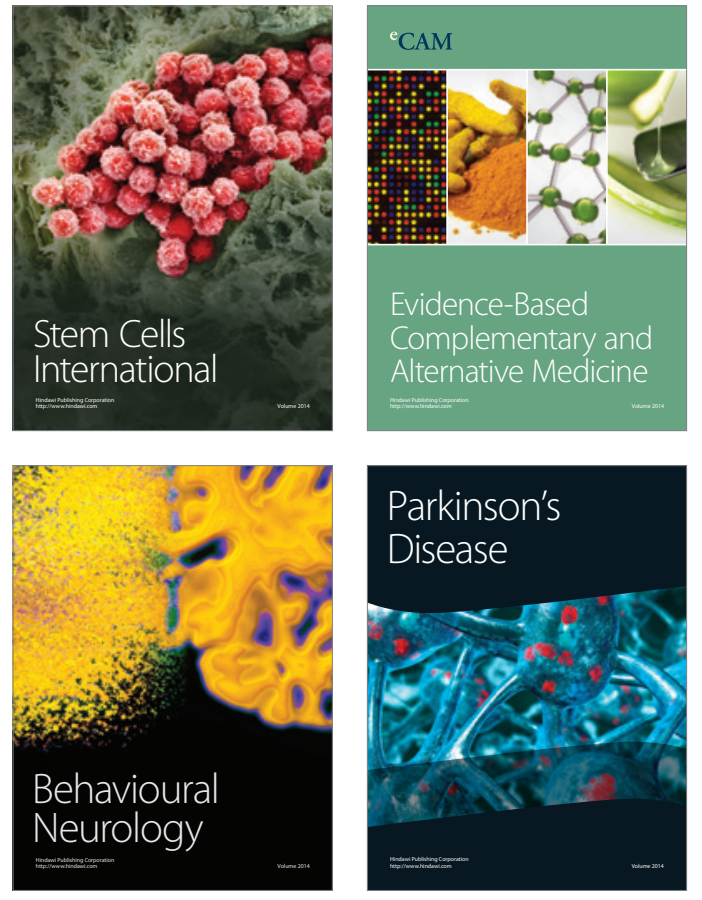
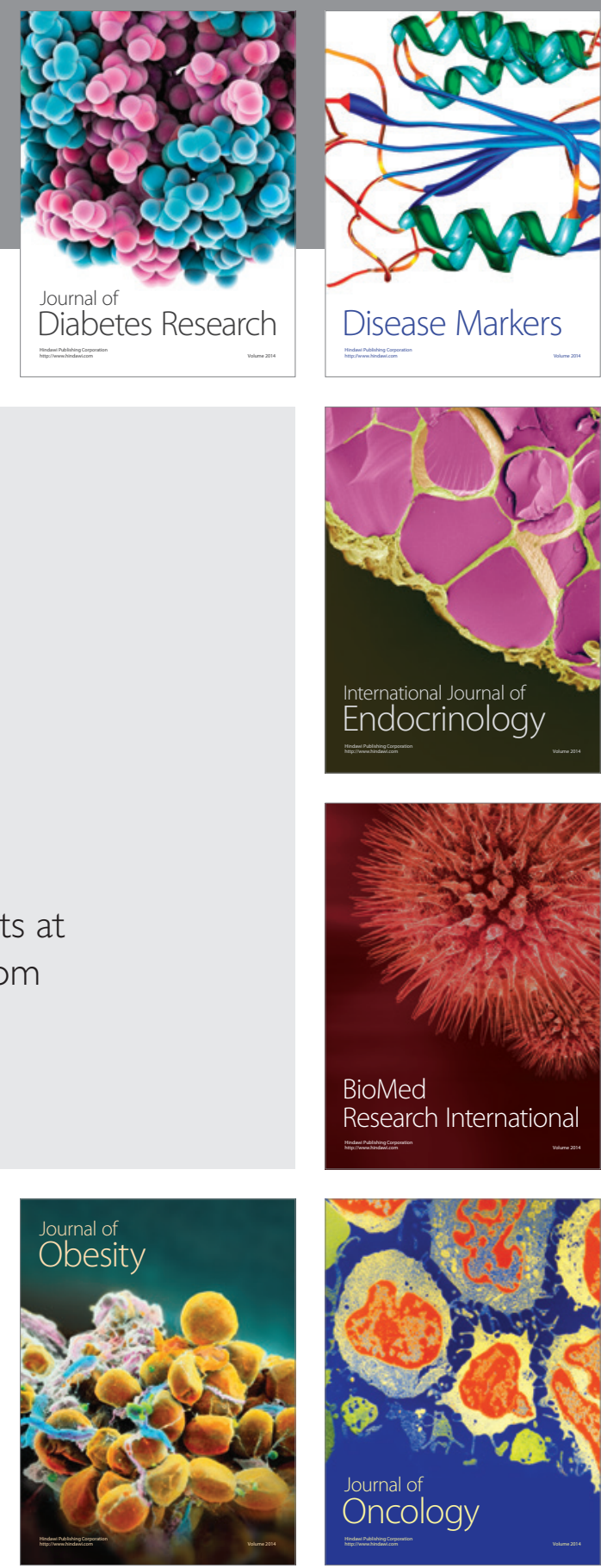

Disease Markers
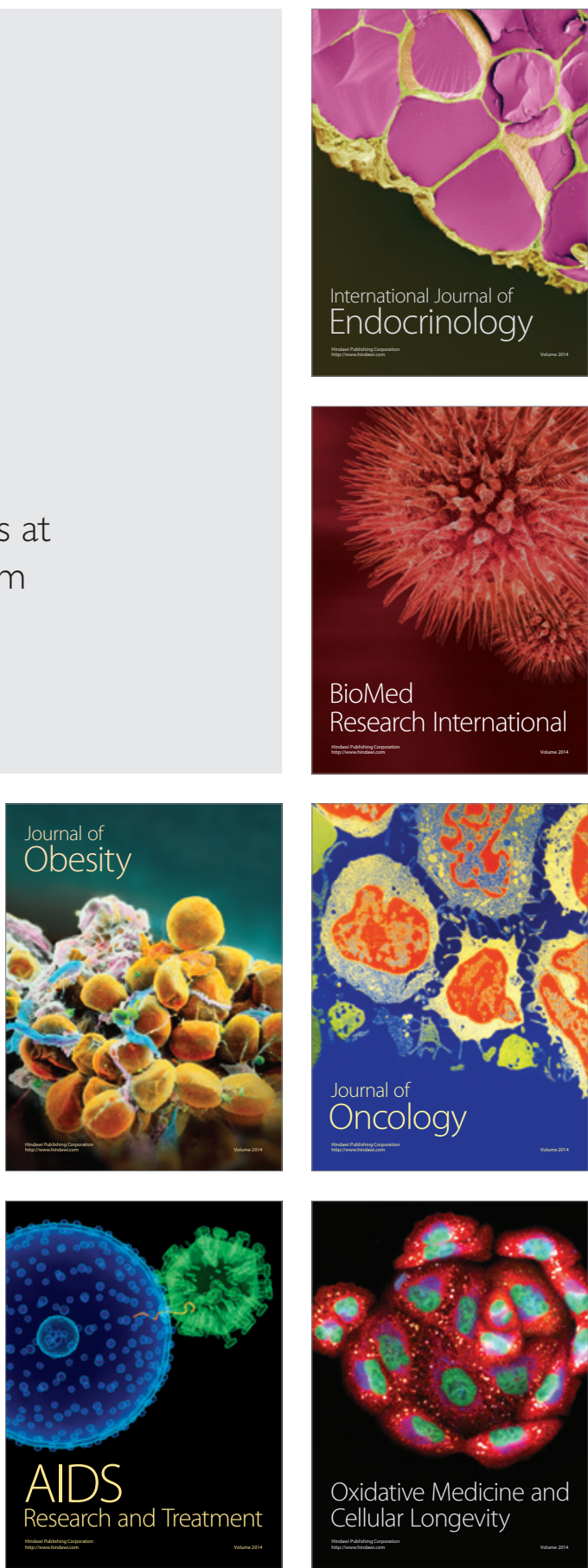